\title{
A New Scheme for Network Selection in Heterogeneous Wireless Network using Fuzzy Logic
}

\author{
NitinGoel \\ Research Scholar, \\ IIIT Allahabad, India
}

\author{
Neetesh Purohit \\ Asstt Prof, \\ IIIT Allahabad, India
}

\author{
B.R.Singh \\ Professor, \\ IIIT Allahabad, India
}

\begin{abstract}
Ensuring seamless and reliable switching across different constituent technologies is the basic requirement of heterogeneous wireless networks. IEEE 802.21 standard is recommended for $4 \mathrm{G}$ networks for coordinating among different mobile network for better services. Still, there exists a scope of further improving its performance by introducing the fuzzy logic concepts in it. The present work aims in this direction where a fuzzy interface system is designed using four different parameters viz. speed of mobile terminal, available bandwidth, received signal strength and number of users. The simulation result show that the number of vertical handoffs in the proposed technique reduced by $27 \%$ as compared to the existing fuzzy approach and by $42 \%$ as compared to the classical approach.
\end{abstract}

\section{Keywords}

Heterogeneous networks, seamless mobility, fuzzy logic, handoff.

\section{INTRODUCTION}

The heterogeneous wireless network is a collection of different wireless technologies like cellular networks, wireless metropolitan area network (WiMax) and wireless local area network (WiFi). Besides traditional handoff (between cells of same technology), a vertical handoff (between cells of different technology) is the primary requirement for the proper functioning of heterogeneous networks. The most desiring features are to maintain best quality of service (QOS) by connecting to most fascinating network among the available networks.

Figure 1 shows the integrated network structure of cellular networks, Wi-Fi and WiMax. In WiFi or WiMax the point of attachment through which a user gets connected is called an access point and in cellular network is termed as base station. The three basic steps in the vertical handover process are network discovery, handover decision and handover execution process [1].

In the traditional cellular system also known as GSM, the threshold comparison of different specific parameter metrics is used to make handover decisions [2]. The inter-system handovers are not only supposed to maintain ongoing connections but also to provide users with better services and to meet their individual requirements. In contrast to horizontal handover, vertical handover considers not only the received signal strength (RSS) but also the service class

Mapping between handoff-in and handoff-out networks [3].The IEEE 802.21 standard defines the basic requirement for heterogeneous mobility. Quality of service is measured with the performance of transmission of data, network technologies and service accuracy [4]. The idea is to provide good connectivity with different network, reduction in call dropping and good quality of service. A cell phone call may be interrupted or drop after an unnecessary handover or incorrect handover. An unnecessary handover decision can degrade the quality of service and can also interrupt the ongoing communication. So it is essential that the handover process execute seamlessly and accurately to improve the quality of service. The multimedia applications like video streaming, VOIP, web browsing requires good quality of service. Therefore it is mandatory to indicate the quality of a vertical handover network selection and the network performance. In the vertical handover quality can be define as reduction in ping-pong effect, reduction in interruption in the ongoing call during handover and enhancing the throughput of the system [5].The scheme presented in this work shows better performance in this regard.

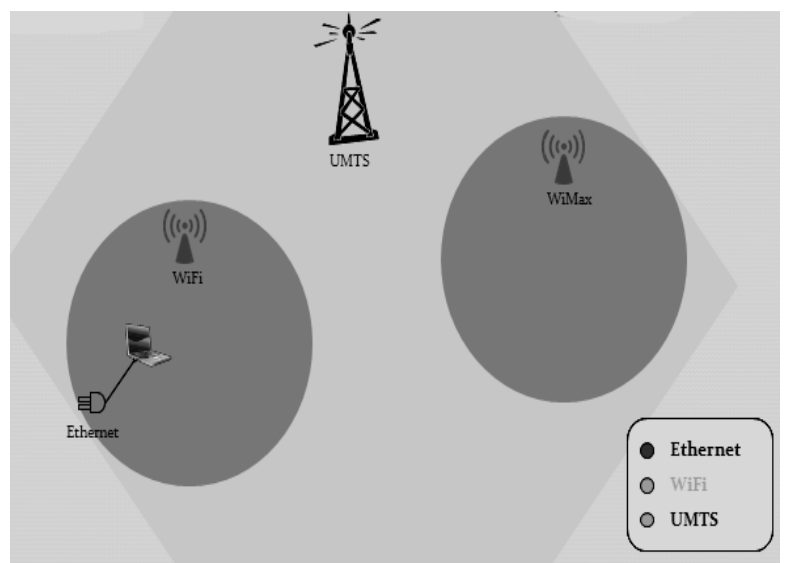

Fig1 Integrated cellular,Wi-Fi and WiMax network

There is variety of architectures, algorithms and schemes for vertical handover network selection have been reported. Some of the algorithms are based on classical method of network selection technique [6] and some are on Markov decision process [7]. Following are the important parameters which are considered in handoff decision in developing the proposed scheme. Fast and seamless handover is a big challenge for next generation IP-based heterogeneous networks which is supposed to support real-time high-speed multimedia applications. These applications requires small handover latency, packet loss and high data-rate transmission [8].Best Network Selection: Realization of seamless handover to the best network selection while considering quality of service and AAAC(Authentication, Authorization, Accounting and Charging).Prioritization of networks becomes more complicated if a potential large number of candidate networks are considered. 
In executing handover decisions, received signal strength (RSS) is not only significant parameter. In order to overcome this drawback, other metrics such as bandwidth availability, RSS variation, quality of service parameters, network conditions, seamlessness etc. must be considered while initiating a handover process. To minimize the number of handovers especially when user is roaming around border of the cell or moving fast is also required. This can also define as ping-pong effect [9].

The paper is organized in four sections. The section II contains the proposed scheme; results are reported in section-III which is followed by discussion. The conclusion is presented in section IV.

\section{PROPOSED FUZZY BASED APPROACH}

For vertical handover there are different approaches exists. In the classical approach, the decision of network selection is based on comparison of received signal strength (RSS) from the access point (AP) of $\mathrm{WiFi} / \mathrm{WiMax}$ network with the signal strength getting from base station of a cellular network when a mobile user approaches the cell boundary every time [10]. The received signal power decreases accordingly with the increase in the distance between a transmitter and a receiver. If a mobile user is in a covered area or in an open plain the received signal strength decreases with the increase in distance between a transmitter and a receiver in the same fashion [11]. The basic concept of fuzzy logic is that of a linguistic variable, that can be defined as a variable whose values are not the numbers. The fuzzy logic may be ideal for this situation because its methodology for computing linguistic variable very well. The linguistic variables are less precise than numbers but it is easy to understand. Computing with linguistic variable enhance the tolerance for imprecision and also lowers the cost of solution [12].

The fuzzy based network selection approach deals with a network selection based on fuzzy theory taking into account some parameters. The parameters are like RSS, speed of mobile terminal and some other factors [8]. After establishing the membership functions, the membership degree is calculated as function of input parameters. In this proposed approach, four input parameters: RSS, velocity of Mobile Terminal, bandwidth available and number of users are considered. The decision of network selection is based on fuzzy approach. Fuzzy approach is chosen because of its ability to handle the non-precise and uncertain data. The fuzzy logic also has the advantage of adding rules in simple language. The steps to implement a FIS system can be explained as first convert real values into a membership functions then write set of if-thenelse rules to develop a fuzzy inference engine there after see the result to produce the real values of the desired output. The proposed technique uses fuzzy inference system (FIS) graphic user interface editor and reviewer to implement all the steps involved in fuzzy system, such as defining membership functions, building the rules and analyzing the results in fuzzy inference engine.
Table 1 Parameters of network selection system

\begin{tabular}{|c|c|l|}
\hline $\begin{array}{c}\text { Parameters } \\
\text { Mobile } \\
\text { terminal }\end{array}$ & $\begin{array}{c}\text { Range } \\
300 \mathrm{~km} / \mathrm{hr}\end{array}$ & $\begin{array}{l}\text { The speed of the mobile terminal } \\
\text { is depending on whether the user } \\
\text { is moving through two-wheeler } \\
\text { or through aeroplane. }\end{array}$ \\
\hline $\begin{array}{c}\text { Available } \\
\text { bandwidth }\end{array}$ & $\begin{array}{c}100-1000 \\
\text { Mbps }\end{array}$ & $\begin{array}{l}\text { This shows the available } \\
\text { bandwidth offered by the } \\
\text { network to mobile user. It } \\
\text { depends on the number of users } \\
\text { which is currently in this } \\
\text { particular network. }\end{array}$ \\
\hline $\begin{array}{c}\text { Receive } \\
\text { Signal }\end{array}$ & $\begin{array}{c}-95 \mathrm{dbm} \text { to } \\
\text { Strength } \\
\text { for mobile } \\
\text { network }\end{array}$ & $\begin{array}{l}\text { This is the threshold value of the } \\
\text { signal strength for the wireless } \\
\text { network. }\end{array}$ \\
\hline $\begin{array}{c}\text { Number of } \\
\text { User }\end{array}$ & $0-50$ & $\begin{array}{l}\text { Depending of the active user the } \\
\text { quality of service provided by } \\
\text { the network varies. }\end{array}$ \\
\hline
\end{tabular}

The fuzzy inference system accepts the values of a particular factor and assigns the computed values to the output according to user-defined rules. The fuzzy inference system is compact and computationally efficient for this type of computation. The fuzzy system is a system which is used adaptive techniques for constructing models and this technique can be used to utilize the membership functions so the fuzzy interface system provides the output for the given input data. Fuzzy inference system works on if-then-else rules. The idea of handover between Wi-Fi to cellular network and cellular to WiFi is quite different. In heterogeneous network as shown in fig-1, the time taken in vertical handover from WiFi to cellular network is important because the delay in the handover process can lead to disconnection of service, whereas the vertical handover from cellular network to WiFi can wait for little longer duration because the network structure is heterogeneous and the base station signal of cellular network is available even in the $\mathrm{WiFi}$ region. Therefore both of these networks have different condition so there must be different set of rules and different threshold values for vertical handover process for cellular to $\mathrm{WiFi}$ and for WiFi to cellular network. These different threshold values have to be use in the process of vertical handover decision for more accurate decision making.

\section{RESULTS AND DISCUSSION}

The proposed technique is simulated in MATLAB environment. The fuzzy based system is designed in fuzzy logic toolbox. This technique need to define some parameters based upon information of the user equipment and the network condition. The parameters used are available bandwidth, speed of mobile terminal, number of user and received signal strength. In these parameters the speed of mobile terminal and RSS are related to source information of user equipment and available bandwidth and number of user are related to source information of the network condition. 


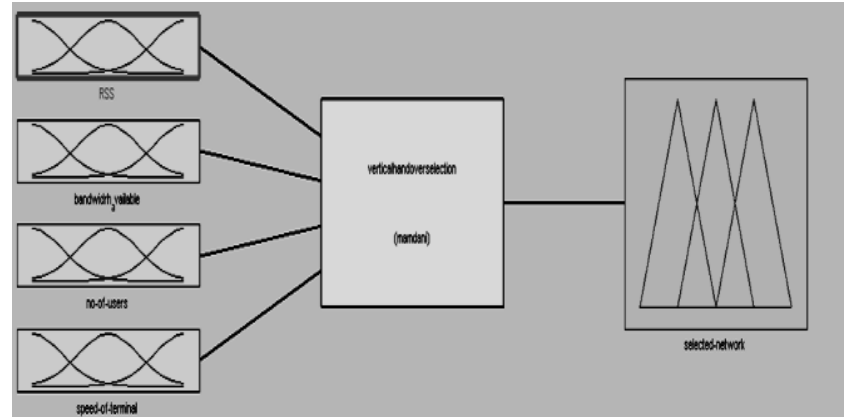

Fig 2.FIS Editor Window of Network Selection

The FIS Editor displays general information about the fuzzy inference system. The editor shows the names of each input variable on the left, and those of each output variable on the right. The FIS Editor handles the issues for the system. In the designing of FIS editor for this technique the four input parameters are used as shown in table 1. After applying rulers the performance of the system can see on rule viewer. Fig 2 show the FIS system used to get the performance. In the left hand four boxes represent the parameters which define in Table 1 in the form of membership function. The box in the middle is set of rule which is also called MADM function and the right box represent the type of network which is required to be selected.

A membership function of the given fuzzy set maps an input value to its appropriate membership value. Fig 3 represent the membership function editor of receive signal strength. According to the range of receive signal strength the division has been done as low, average and good. The Membership Function Editor is used to define the type of membership functions associated with each variable. The fuzzy logic toolbox in matlab contains 11 built-in membership function types.

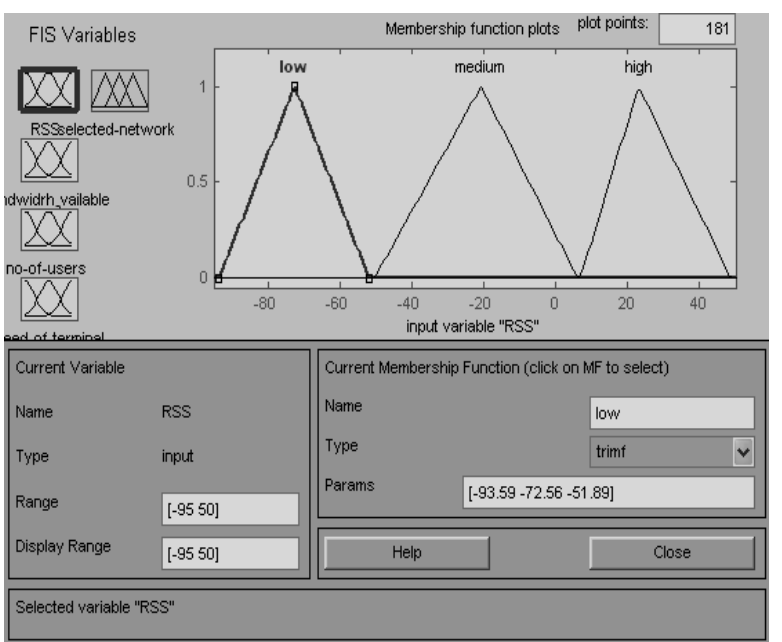

Fig 3.Membership function of receive signal strength

The triangular membership function (trimf) is used to represent the different range of the parameters of this network selection technique. This function is a collection of three points forming a triangle.

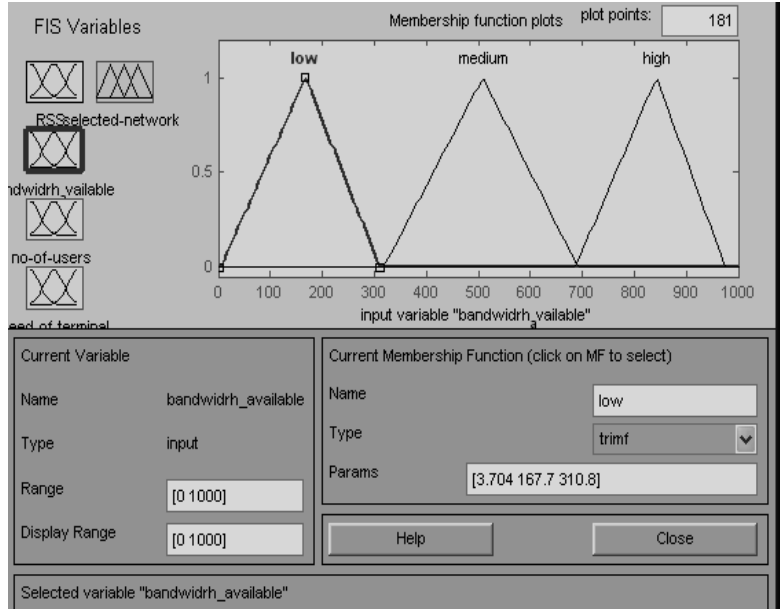

Fig 4.Membership function of Available Bandwidth

Fig 4 represents the membership function editor of available bandwidth. In this also the division of range of available bandwidth has been done as low, medium and high.

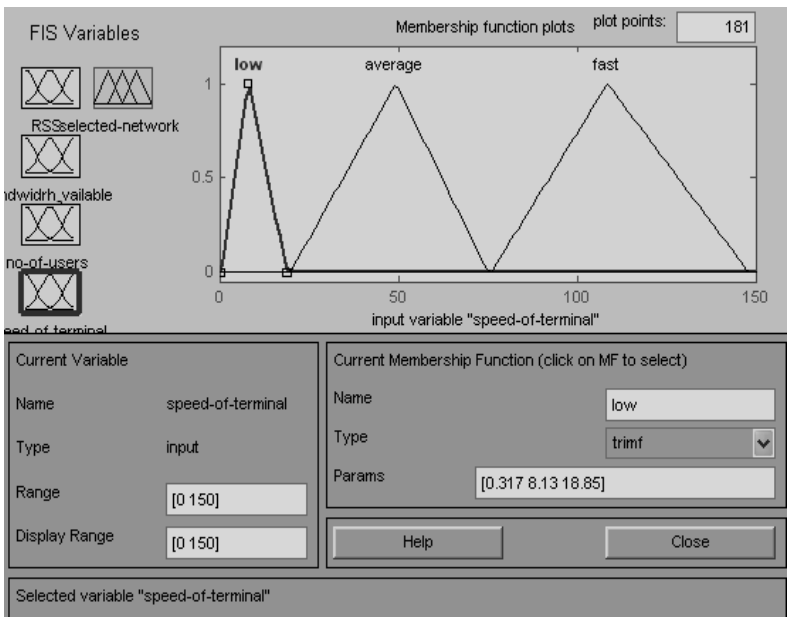

Fig 5.Membership function of velocity of mobile terminal

Fig 5 represents the membership function editor of velocity of mobile terminal. The range of velocity of mobile terminal is divided as slow, medium and fast.

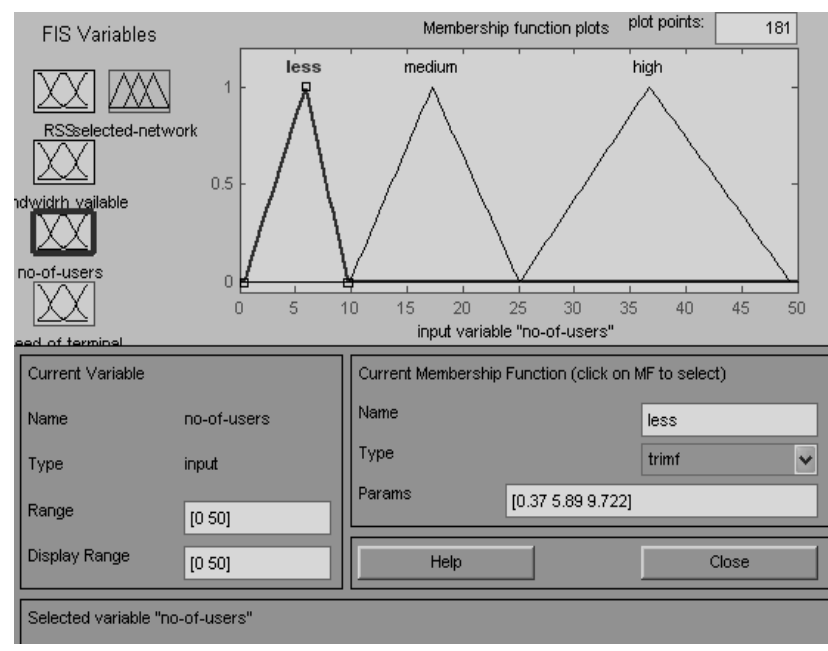

Fig 6.Membership function of number of users 
Fig 6 represents the membership function editor of number of users. The range of number of users is also divided into less, average and dense according to the active users connected with the network at a particular time.

Rules in the fuzzy interface system works as a condition in which the system has to perform the task. The importance of the fuzzy logical reasoning is the fact that it is a superset of standard Boolean logic. In other words, if it keeps the fuzzy values at their extremes, standard logical operations will hold. In the fig 7 it explains the different logic in which the system has to measure the performance of the system. Fuzzy sets and fuzzy operators are operating on if-then rules. These if-then rule statements are logically used to formulate the conditional statements that comprise the output of the fuzzy system. In this system, the input of an if-then rule is the current value for the input variable and the output is the entire fuzzy set.

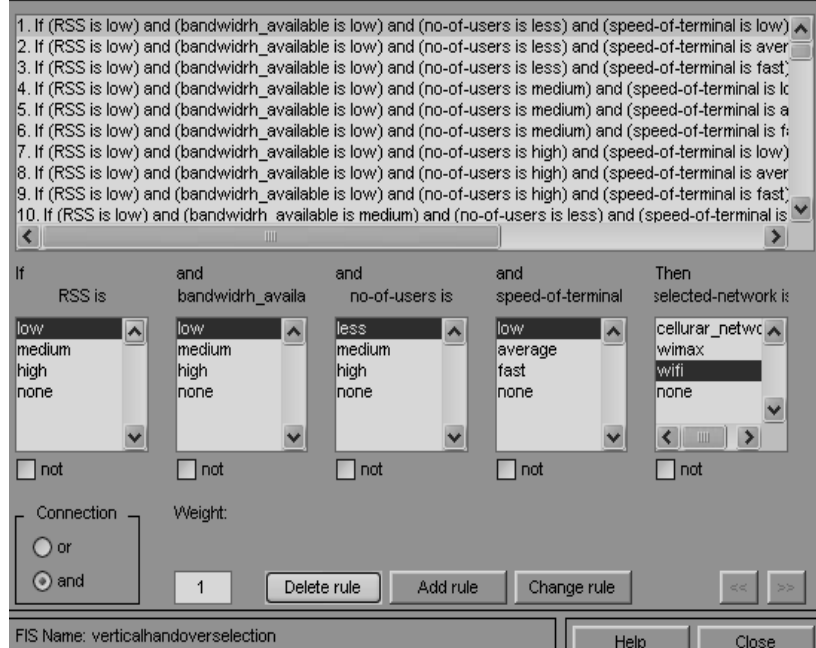

Fig 7 Rule Editor of Network Selection Interface system

Fig 7 also defines the rules which are a function to implement this experiment. This function is implemented on input which is taken in fig 2 to get the output. The if-then rule can interpret as first fuzzifying the input and applying any necessary fuzzy operators and then applying that result to the consequent also known as implication. In the case of multi-valued, if-then rules do not present much difficulty. Interpreting the if-then rules is the three step process. The first step resolves all fuzzy statements in the antecedent to a degree of membership. The second step apply fuzzy operator to multiple part antecedents. In the third step use the degree of support for the entire rule to shape the output fuzzy set. The input of a fuzzy rule assigns an entire fuzzy set to the output. This fuzzy set represented by a membership function that is chosen to indicate the qualities of the desired system.

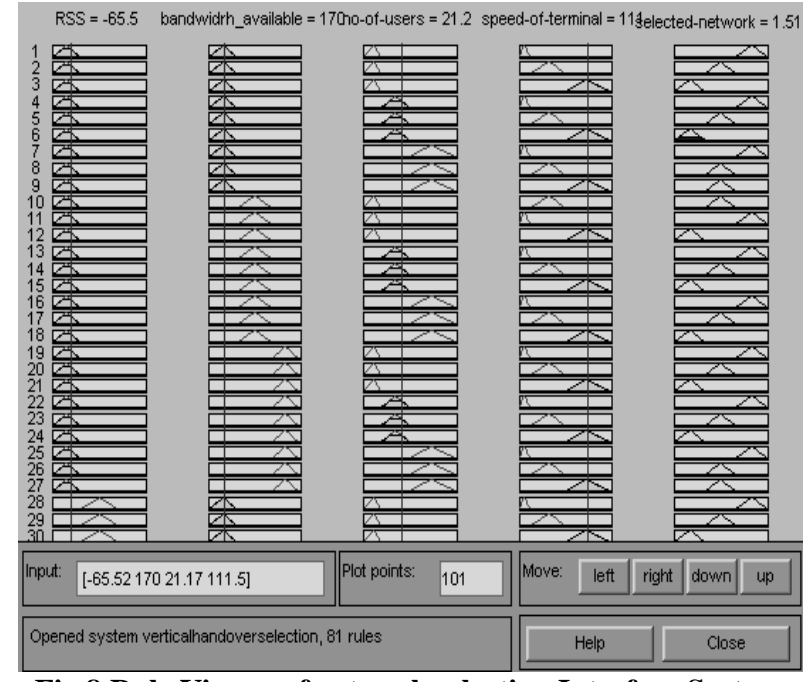

Fig 8.Rule Viewer of network selection Interface System

In general only one rule alone is not that much effective. Two or more rules are required that get the accurate result or to make any prediction. The output of each rule is a fuzzy set. The output fuzzy sets for each rule are then aggregated into a single output fuzzy set. Fuzzy Inference Systems shows how the whole process works from beginning to end for a particular type of fuzzy inference system called a Madm function. In fig 10 the Rule Viewer displays the whole fuzzy inference process. It is based on the fuzzy inference diagram.

Fig 8 shows the output of the different input values. In this figure the line passing from each first four columns show the possibility of the input data available and the line in the fifth column show the output performance range of that input values. The numerical value of the performance shows possibility of the performance range which are cellular, WiFi and WiMax.

The three-dimensional output curve represents the mapping between different input parameters and showed the performance of the system. Because this system has four-input one-output parameters, this cannot see the entire mapping in one plot. This problem can be solved by selecting any two inputs and any one output.

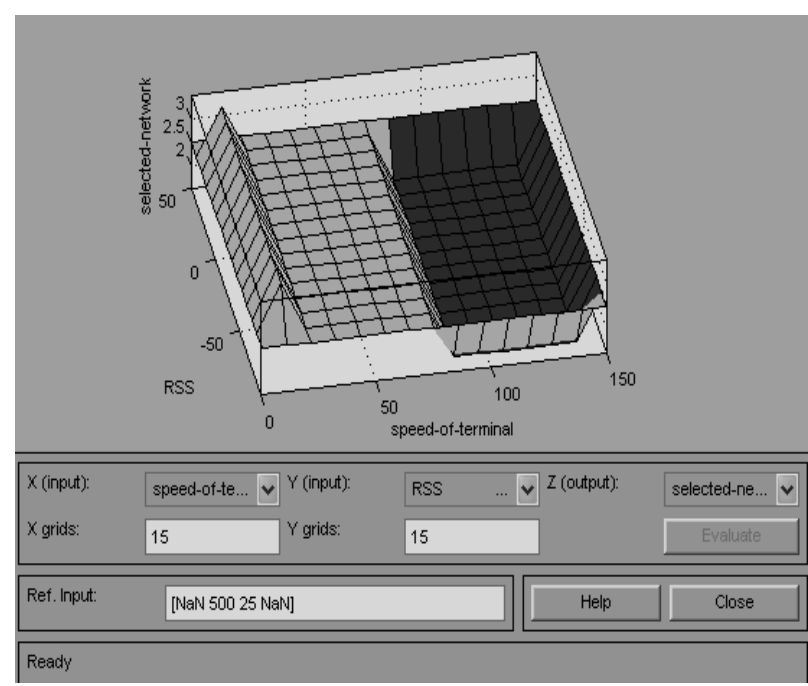

Fig 9.Surface Viewer between Receive Signal Strength and speed of mobile terminal 
The three-dimensional output graph has any two of the inputs parameters and the remaining two of the inputs remain constant. Fig 9 represents the graph between receive signal strength, speed of mobile terminal and selected network.

The comparison of classical approach, existing fuzzy approach and the proposed fuzzy approach has been made by measuring the number of vertical handoffs. Number of handoff is an important parameter when comparing the performance of different handoff techniques. The number of vertical handoffs calculated in a simulated environment developed in c language. It is desired to have minimum number of handoff and all the requirements need to be fulfilled. Total simulation time is $100 \mathrm{~s}$ for each approach. The results show after the comparison of proposed approach with existing fuzzy approach and the classical approach, that in the proposed technique the number of handoff reduces while all the user requirements are fulfilled. This can be shown in Fig. 10 the proposed fuzzy based technique decides handoff much less times then the existing fuzzy based approach and classical approach respectively.

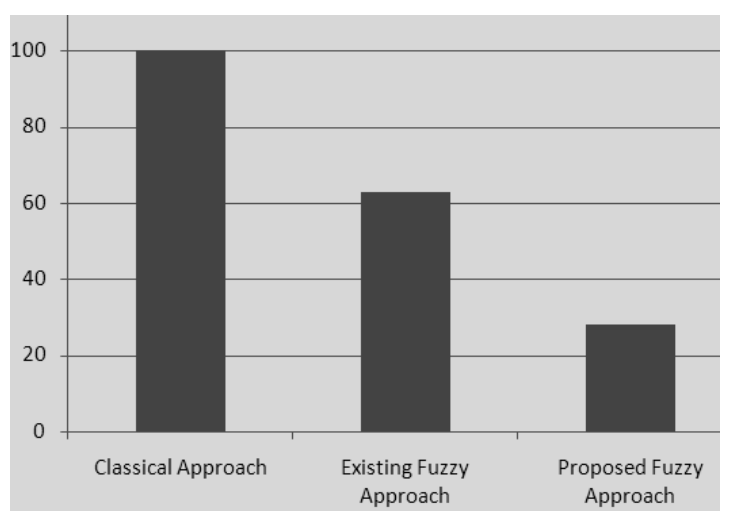

Fig 10.Performance of each handoff decision technique

\section{CONCLUSION}

A new scheme for network selection is proposed for the vertical handoff in heterogeneous wireless network. This approach ensures the seamless mobility in the integration of WiFi, WiMax hotspots and cellular networks in a better way as compared to IEEE 802.21. However further optimization should be done for better resource utilization, latency minimization. The future aspect of this technique is to enhance the mobility of the user and fulfill the expectation of anywhere, anytime connectivity with the network. The network selection is only one aspect and the next process is to execute the process of handoff.

\section{ACKNOWLEDGMENT}

The authors wish to express their sincere thanks to director, IIIT Allahabad, for the support and encouragement.

\section{REFERENCES}

[1] Fang Zhu and Janise McNair Multiservice Vertical Handoff Decision Algorithms Accepted 26 May 2006 Hindawi Publishing Corporation.

[2] Ahmed Hasswa A seamless context-aware architecture for fourth generation wireless networks received 15May 2006 Accepted 20 January 2007 published online 12 April 2007 Springer science-business BV 2007

[3] Pravin Pawar-Context-Aware Middleware Architecture for Vertical Handover Support to Multi-homed Nomadic Mobile Services- $S A C$ '08, March 16-20, 2008, Fortaleza, Ceará, Brazil, ACM.

[4] Yaw Nkansah-Gyekye, Vertical Handoff Decision Algorithm for UMTS-WLAN, the 2nd International Conference on Wireless Broadband and Ultra Wideband Communications (Aus Wireless 2007).

[5] Enrique Stevens-Navarro and Vincent W.S. Wong, Comparison between Vertical Handoff Decision Algorithms for Heterogeneous Wireless Networks, 2006 IEEE.[6]. Mingxin Li, A Multi-step Vertical Handoff Mechanism for Cellular Multihop Networks, PM2HW2N'07, October 26, 2007, Chania, Crete Island, Greece. Copyright 2007 ACM.

[6] Anita Singhrova, A Review of Vertical Handoff Decision Algorithm in Heterogeneous Networks, MC'07 (Mobility'07), September 10-12, 2007, Singapore. Copyright 2007 ACM.

[7] Inwhee Joe, Sungchan Hong College of Information and Communications Hanyang University Seoul, Korea A Mobility-based Prediction Algorithm for vertical handover in hybrid wireless network 2010.

[8] A. Singhrova, N. Prakash "Vertical handoff decision algorithm for improved quality of service in heterogeneous wireless networks" IET Commun., 2012, Vol. 6, Iss. 2, pp. 211-223.

[9] K. Vasu, Sumit Maheshwari, Sudipta Mahapatra, C. S. Kumar "QoS Aware Fuzzy Rule Based Vertical Handoff Decision Algorithm for Wireless Heterogeneous Networks" 978-1-61284-091-8/11/\$26.00 @2011 IEEE.

[10] Carlos Ramirez-Perez and Victor M. Ramos R "A QoS hierarchical decision scheme for vertical handoff" 2012 8th International Caribbean Conference on Devices, Circuts and Systems (ICCDCS).

[11] TAO Yang, PENG Rong "A Fuzzy Logic Vertical Handoff Algorithm with Motion Trend Decision" 2011 the 6th International Forum on Strategic Technology. 